\title{
Schmetterlinge und Brennnesseln - Kinder im Vorschulalter entdecken die Nahrungsvorlieben einheimischer Schmetterlinge
}

\author{
Melanie Willführ, Ulrike Brunken, Christina Hoede, Katharina Kamm, \\ Jutta Lehmann \& Ina Pfaff
}

\begin{abstract}
A new program of the educational service „Kinder im Garten“ is outlined: subsequent to the new exhibition featuring tropical butterflies, children can explore the nutritional requirements of local butterflies, using the stinging nettle as an example.
\end{abstract}

\section{Zusammenfassung}

Ein neues Bildungsangebot der Bildungseinrichtung „Kinder im Garten“ zum Thema Schmetterlinge wird vorgestellt: Anknüpfend an die Ausstellung zu tropischen Schmetterlingen wird der Nahrungsbedarf einheimischer Schmetterlingsraupen am Beispiel der Brennnessel mit den Kindern erforscht.

Schmetterlinge üben auf Kinder im Vorschulalter eine große Faszination aus. Die Schmetterlingsschau im Blüten- und Schmetterlingshaus ist Anlass für ein neues Themenangebot der Bildungseinrichtung „Kinder im Garten“, einer Kooperation zwischen Palmengarten und Kita Frankfurt, welche sich mit ihren Angeboten speziell an Kinder im Alter von 3-6 Jahren richtet.

Ziel wird es sein, die Kinder neben der Begeisterung für die farbenfrohe Welt der Schmetterlinge auch für deren Lebensweise und Lebensgrundlagen zu sensibilisieren und Möglichkeiten zu zeigen, wie jeder zu deren Erhalt beitragen kann.

Meist ist den Kindern der Lebenszyklus der Schmetterlinge bekannt, insbesondere der Gegensatz zwischen unscheinbarer Raupe und buntem Falter. Kinder, die den Zyklus noch nicht kennen, erforschen anhand von Fotos und kleinen Modellen die Abfolge Ei-Raupe-Puppe-Falter. Aber auch auf Fragen, wovon sich Raupen und Falter ernähren, werden gemeinsam Antworten gesucht. Der Bilderbuchklassiker „Die kleine Raupe Nimmersatt" bietet den Kinder viele Ideen und regt zu weiteren Überlegungen an. Hier frisst sich die Raupe durch allerlei vor allem bei Menschen beliebte Nahrungsmittel wie Obst und Schokoladenkuchen und zum Schluss auch ein Blatt, bevor sie sich verpuppt und schließlich in einen Falter verwandelt.
Daran anknüpfend kann man den Kindern Brennnesselblätter zeigen und die Frage stellen, ob auch sie für Schmetterlingsraupen eine gute Nahrung sein können - auf den ersten Blick erscheint dies unwahrscheinlich: Wer hat noch nicht eine eher unangenehme Bekanntschaft mit einer Brennnessel gemacht? Den meisten ist die unfreiwillige Berührung in schmerzhafter Erinnerung. Kinder werden gewarnt, der Brennnessel lieber nicht zu nahe zu kommen. Aber was steckt eigentlich hinter dieser Pflanze mit ihren zahlreichen Brennhaaren?

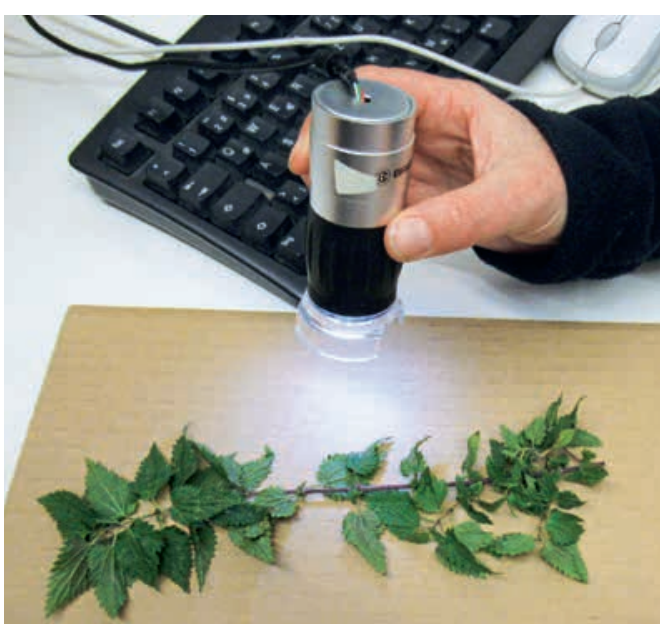

Abb. 1: Mit Hilfe des digitalen Handmikroskops kann mit mehreren Kindern zugleich ein großer Brennnesselspross untersucht und am Bildschirm betrachtet werden. (Foto: Team „Kinder im Garten“) 


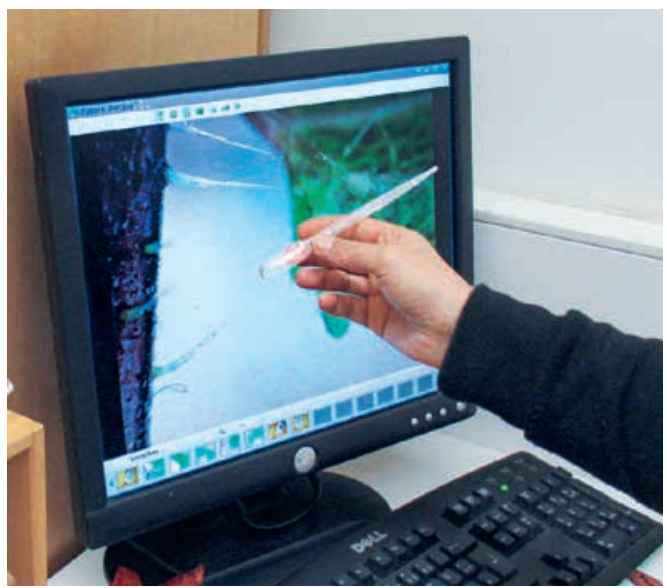

Abb. 2: Am Bildschirm können der Aufbau eines Brennhaares und einer Pipette direkt verglichen werden. (Foto: Team „Kinder im Garten“)

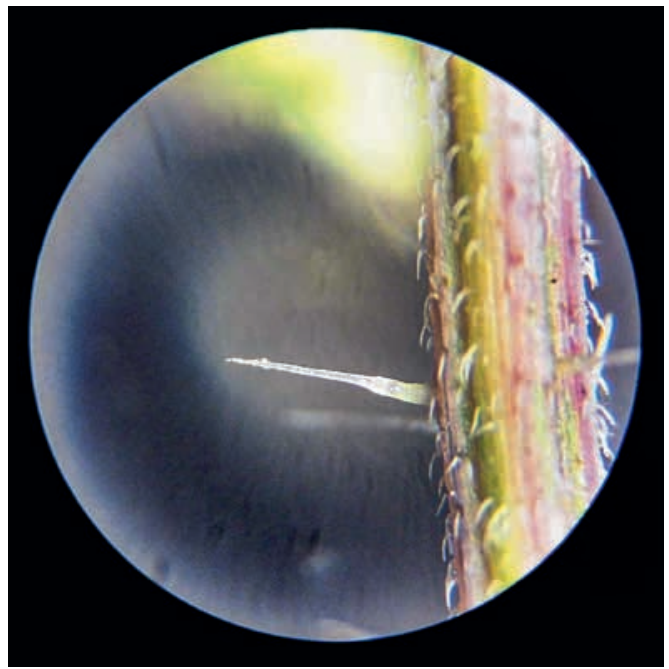

Abb. 3: Aufbau eines Brennhaares. (Foto: U. BRUnken)

Bislang erforschen die Kinder dies noch zum Thema „Wehrhafte Pflanzen“, aber nach der Eröffnung des Blüten- und Schmetterlingshauses im Palmengarten wird diese Forschungsaufgabe auch Programmbestandteil des Bildungsangebots „Schmetterlinge“ sein.

Äußerst vorsichtig, aber sehr neugierig, untersuchen die Kinder Stängel und Blätter auf ihre Brennhaare. Diese sind auch mit bloßem Auge zu sehen. Unter dem digitalen Handmikroskop (Abb. 1) wird in der Vergrößerung der Aufbau

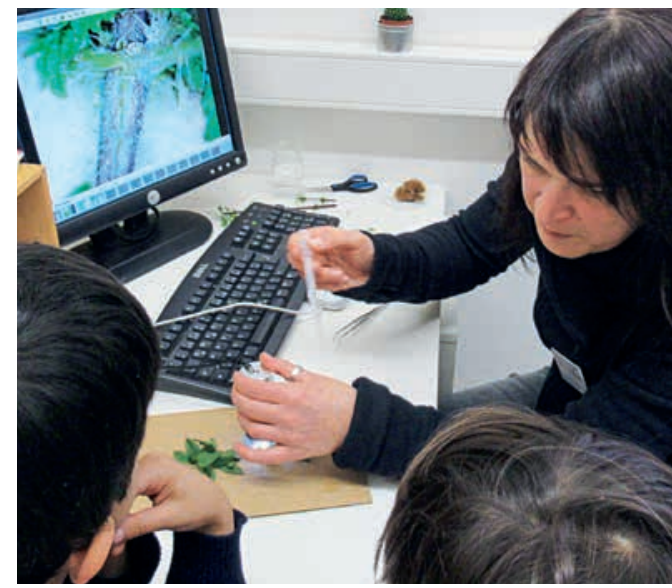

Abb. 4: Probeweise wird versucht, ob auch das Wasser aus der Pipette brennt. (Foto: Team „Kinder im Garten“)

eines Brennhaars genauer sichtbar - er sieht dem einer Pipette ähnlich (Abb. 2): Ein relativ langes einzelliges Haar sitzt mit einer breiteren Basis in einem mehrzelligen Sockel und geht an seiner Spitze in ein kleines schräg sitzendes Köpfchen über (Abb. 3). Sogar eine durchsichtige Flüssigkeit kann man manchmal in den Brennhaaren erkennen. Diese enthält u. a. Ameisensäure und Histamine, welche die brennenden und juckenden Hautquaddeln verursachen. Wie gelangt nun die Flüssigkeit in die Haut? Unterhalb der köpfchenförmigen Spitze eines jeden Brennhaares befindet sich eine verkieselte Sollbruchstelle, die bei Berührung abbricht, so dass die Spitze wie eine Kanüle in die Haut sticht und sich die Flüssigkeit sogar mit leichtem Überdruck in die Haut entladen kann.

Welchen Vorteil hat die Brennnessel dadurch? Den Kindern ist klar: Die Pflanze wehrt sich. Das ist ein Schutz vor Fressfeinden. Mithilfe einer mit Wasser gefüllten Pipette wird den mutigen Kindern der Mechanismus der Brennhaare auf der Hand demonstriert (Abb. 4). Brennt auch das Wasser auf der Haut? „Nein“ ist die einstimmige Antwort und sorgt für allgemeine Erheiterung.

Mit der gemeinsamen Betrachtung von Fotoabbildungen (Kleiner Fuchs, Aglais urticae, Abb. 5) erschließt sich den Kindern die Lösung der Fragestellung. Dir Brennnessel ist eine wichti- 


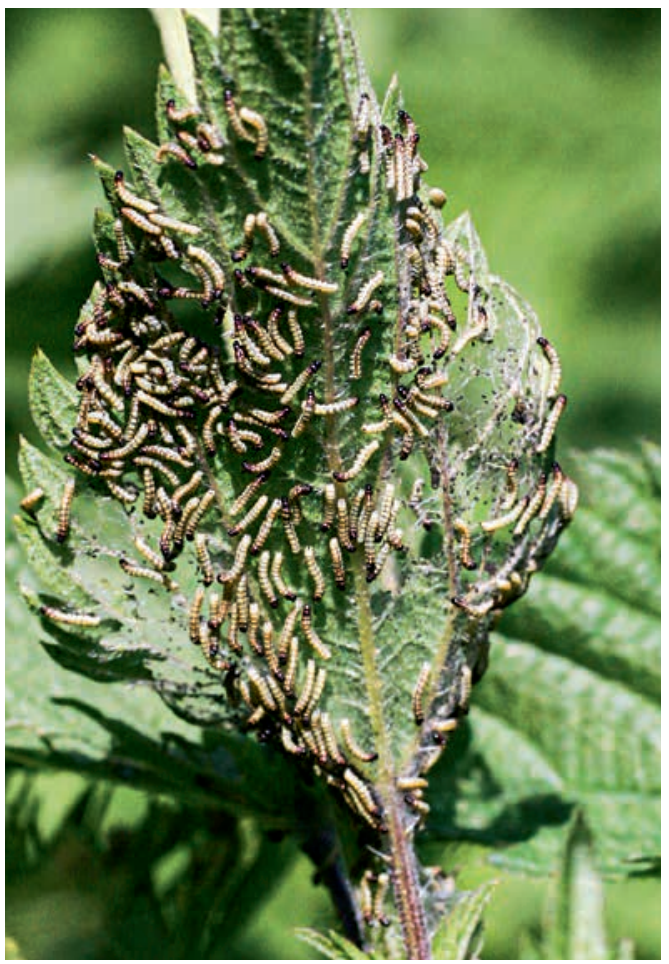

Abb. 5: L1-Räupchen des Kleinen Fuchs.

(Foto: A. Westenberger)

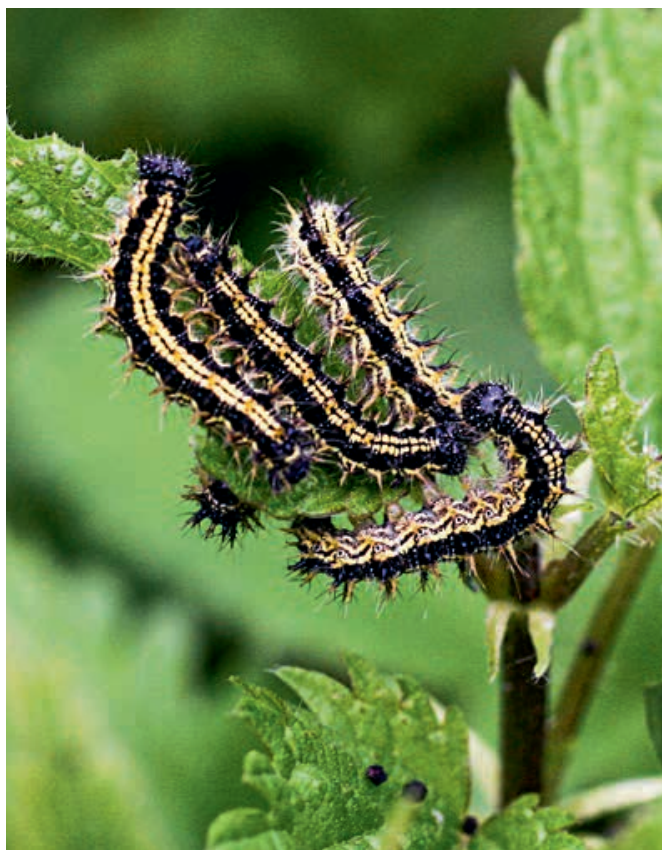

Abb. 7: Halberwachsene Raupen des Kleinen Fuchs. (Foto: A. Westenberger)

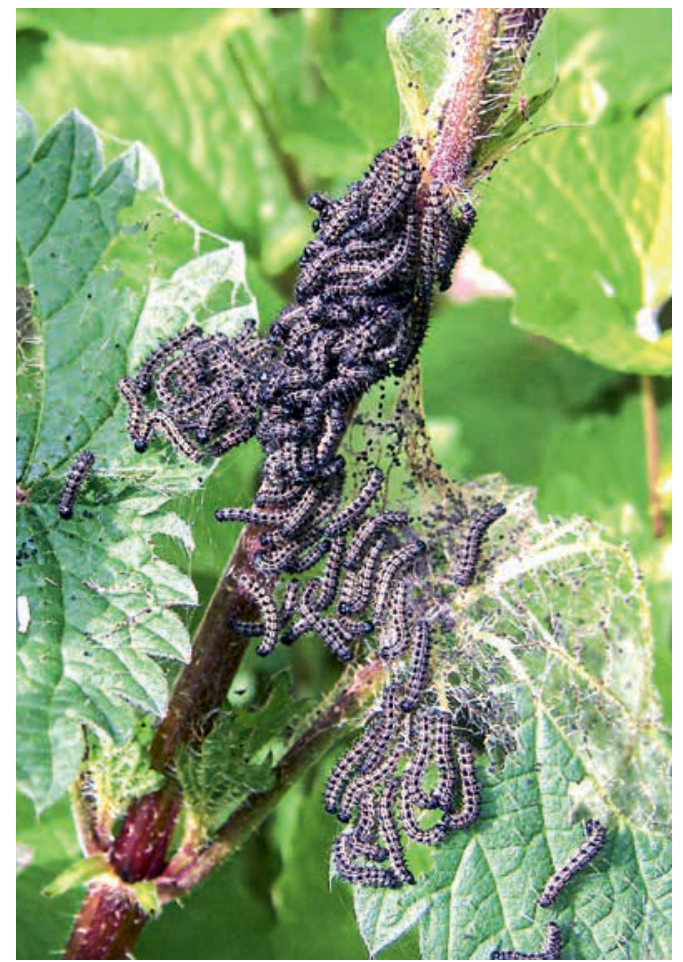

Abb. 6: L2-Jungräupchen des Kleinen Fuchs.

(Foto: A. Westenberger)

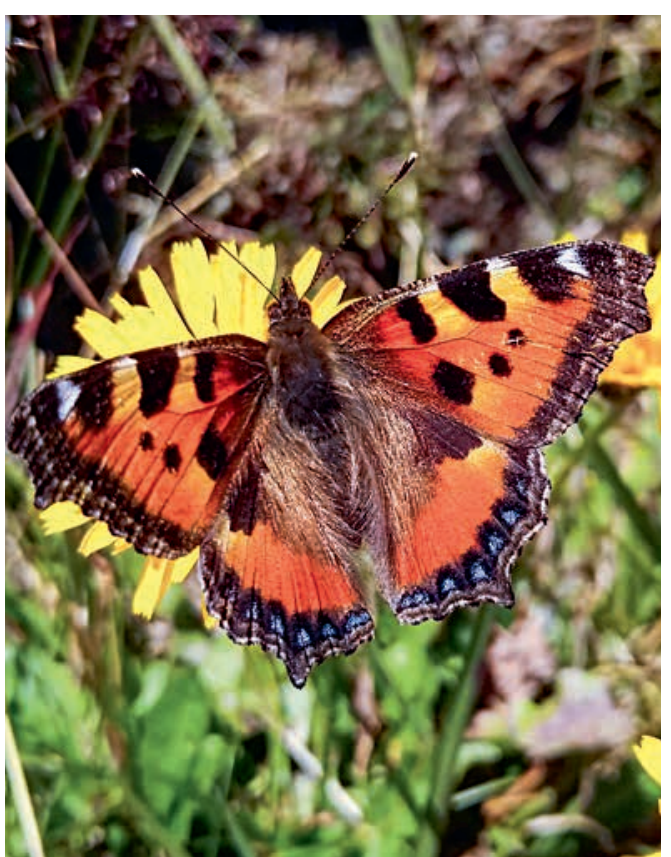

Abb. 8: Kleiner Fuchs. (Foto: H. Steinecke) 


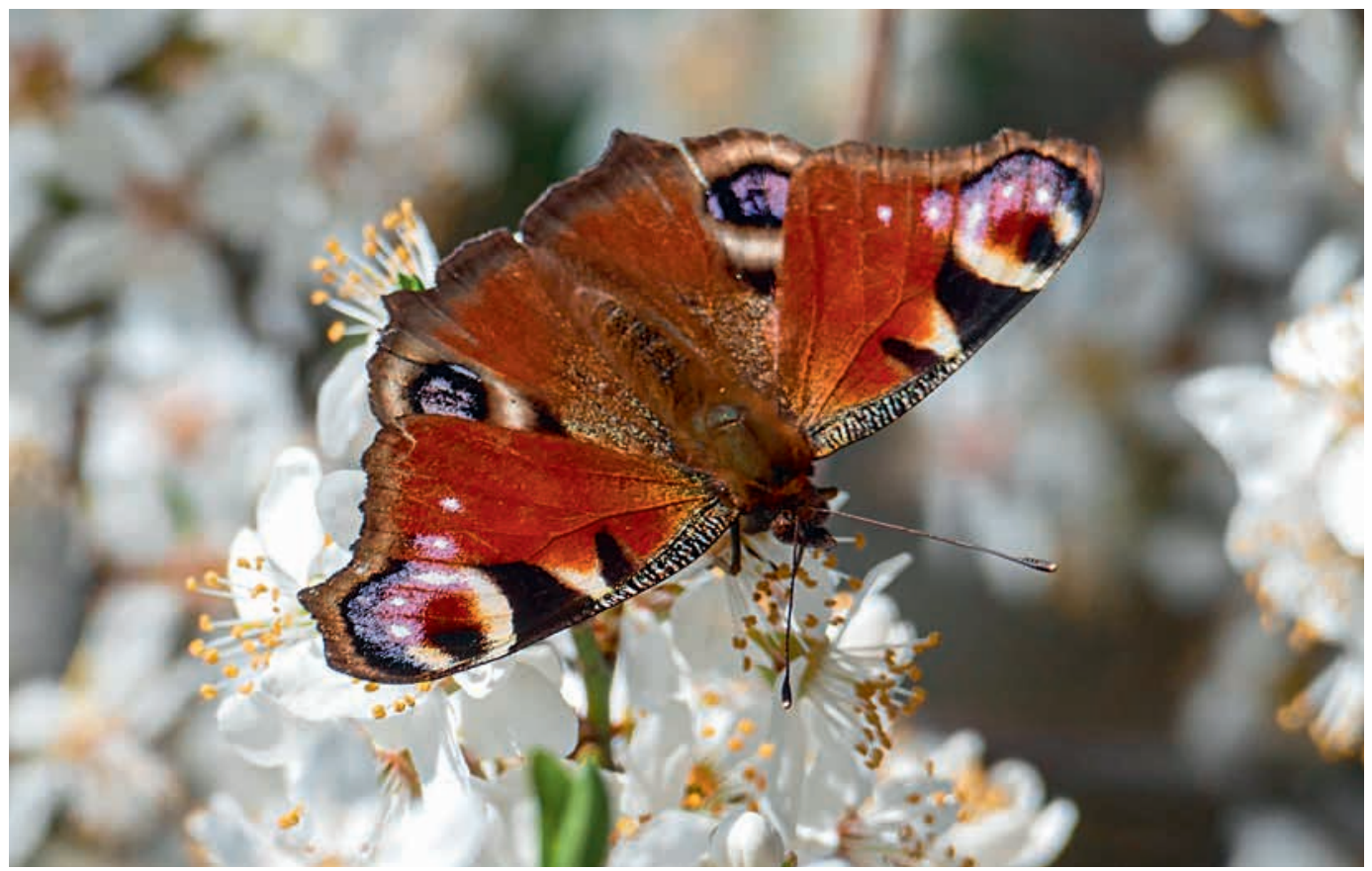

Abb. 9: Tagpfauenauge. (Foto: H. STEINECKe)

ge Futterpflanze für viele Raupen einheimischer Schmetterlinge, und nicht Kuchen, Wurst oder Käse, wie in dem bekannten Buch.

Die Falter ernähren sich im Gegensatz dazu vor allem von zucker- bzw. energiereichem Blütennektar oder anderen süßen Pflanzensäften, die sie mit ihrem Rüssel aufsaugen.

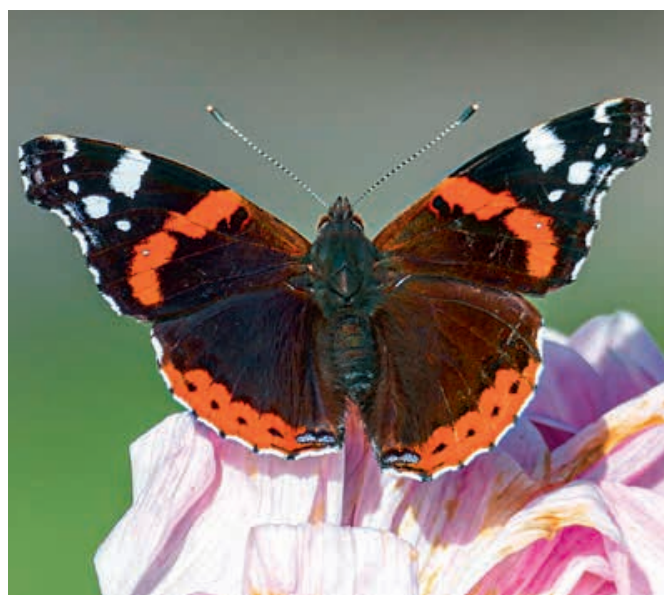

Abb. 10: Admiral. (Foto: H. Steinecke)
Trotz der sehr wehrhaften Eigenschaften der Brennnessel gibt es ca. 30 bis 50 Schmetterlingsarten, die sie im Raupenstadium als Futterpflanze nutzen, zehn davon sogar ausschließlich. Ihnen scheinen die Abwehrmechanismen nichts anhaben zu können. Sie ziehen sogar ihren Nutzen daraus, da die Brennnessel nicht nur sich selbst, sondern auch den Schmetterlingseiern, Raupen und Puppen Schutz vor Fressfeinden bietet. Andere Arten können dagegen auf andere Pflanzen als Nahrung spezialisiert und angewiesen sein.

Zu den vier häufigsten Arten zählen neben dem Kleinen Fuchs auch das Tagpfauenauge (Abb. 9), der Admiral (Abb. 10) und das Landkärtchen. Vorzugsweise benutzen sie für die Eiablage Brennnesseln in halbschattiger oder sonniger Lage. Auch die Raupen des recht häufigen Distelfalters, der bei uns etwa im Mai und Juni als Wanderfalter aus dem Süden zu uns einfliegt, fressen bevorzugt Brennnesseln (Abb. 11).

$\mathrm{Zu}$ unserer Bildungseinrichtung „Kinder im Garten“, die sich im Haus Leonhardsbrunn befindet, gehört ein Schulgarten, in dem Obst 
und Gemüse sowie nektarreiche Pflanzen wachsen (Abb. 12). Eine bunte Pflanzenmischung lockt in den Sommermonaten viele verschiedene Schmetterlinge und andere Insekten an. Auch der Brennnessel als wichtiger Futterpflanze wird dort Raum gegeben. Zwischen Gemüsebeet und Hauswand findet man die Große Brennnessel (Urtica dioica). Der sonnige bis halbschattige Standort ist für die Schmetterlinge sehr attraktiv und wird gerne angenommen. Ein kleiner aber wichtiger Beitrag, um Artenvielfalt zu erhalten und den Kindern das Erforschen einer so „wehrhaften“ und auch schützenden Pflanze zu ermöglichen.

\section{Anschrift der Autorinnen}

Melanie Willführ, Ulrike Brunken, Christina Hoede, Katharina Kamm, Jutta Lehmann \& Ina Pfaff, Palmengarten Frankfurt, Siesmayerstraße 61, 60323 Frankfurt; E-Mails: melanie.willfuehr@stadt-frankfurt.de, ulrike.brunken@stadt-frankfurt.de, christina.hoede@ stadt-frankfurt.de, katharina.kamm@stadt-frankurt.de, jutta. lehmann@stadt-frankfurt.de, ina.pfaff@stadt-frankfurt.de

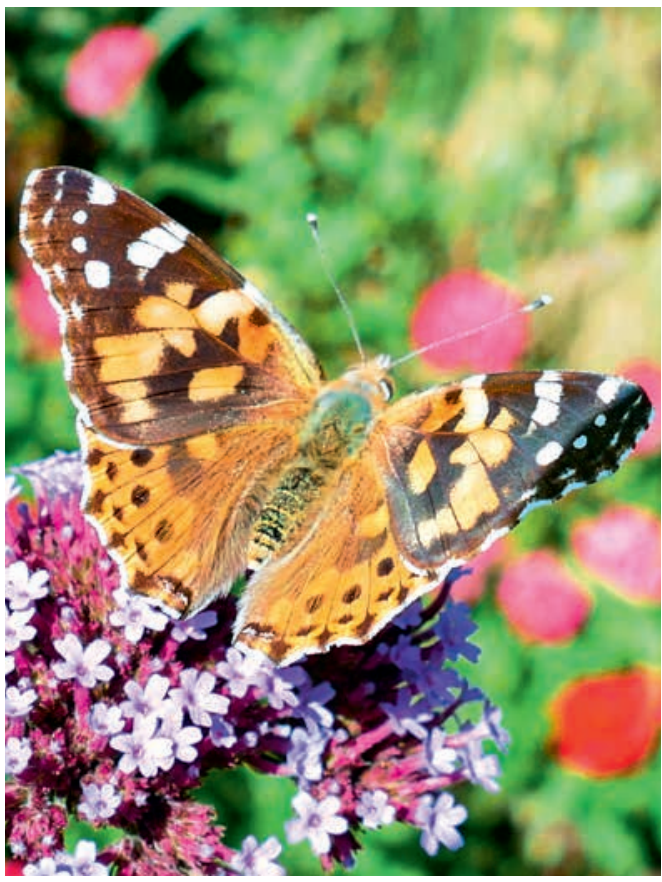

Abb. 11: Distelfalter (Foto: H. STeinecke)

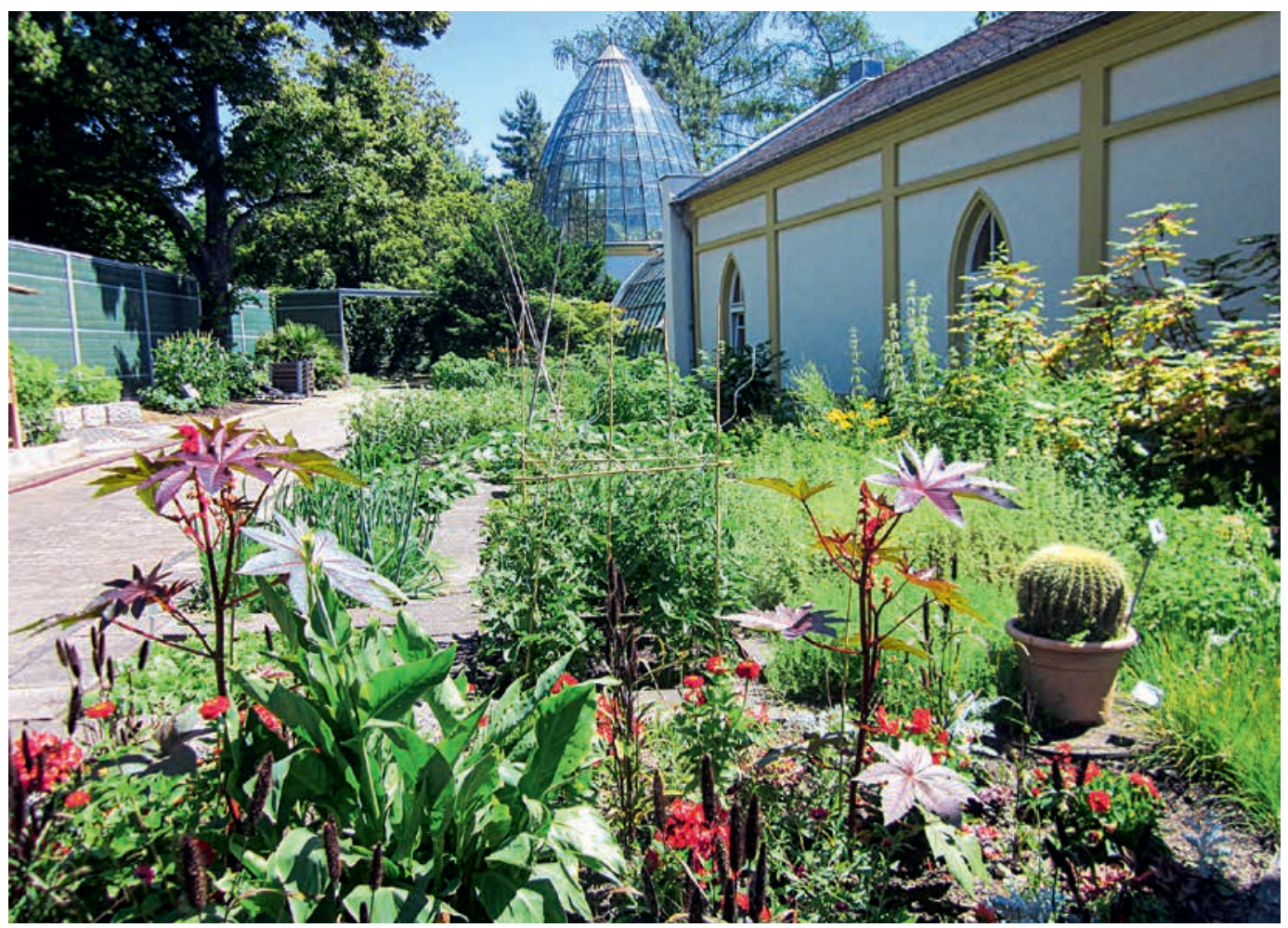

Abb. 12: Garten am Haus Leonhardsbrunn mit Blumenvielfalt und auch Brennnesseln. (Foto: U. BRUNKEN) 